\title{
NITROGEN GAS BEHAVIOR IN SAFETY INJECTION TANK WITH FLUIDIC DEVICE AND EFFECT ON CORE COOLING
}

\author{
YOUNG SEOK BANG \\ Korea Institute of Nuclear Safety, Daejeon, South Korea.
}

\begin{abstract}
A method is developed to determine the hydraulic resistance in Safety Injection Tanks (SIT) with Fluidic Device (FD), which is to be used in the system thermal-hydraulic calculation of Large Break Loss-Of-Coolant Accident (LBLOCA) of Advance Power Reactors 1400 (APR1400). From the overall loss factor estimated from the SIT blowdown test data, the loss factor through the standpipe flow path and the one through the connecting holes of FD are determined from the hydrodynamic consideration. Also, a range of uncertainty of the hydraulic resistances is obtained by confirming that the trend of the SIT test data is covered by the results from the calculations of the test sequence using the set of lower bound K-factors and the set of upper bound K-factors, respectively. Nitrogen release during the blowdown test is discussed in terms of its amount and timing. Implementing the K-factors and the related modelling scheme of SIT, a LBLOCA of APR1400 is calculated using a system thermal-hydraulic code, MARS-KS 1.4. Fuel cladding response and nitrogen gas intrusion to the core are investigated. Sensitivity study is conducted to support to address the effect of uncertainty of SIT/FD to fuel cladding thermal response.

Keywords: ECCS bypass, hydraulic resistances of standpipe and fluidic devices, MARS-KS code, nitrogen gas behavior, safety injection tank with fluidic device
\end{abstract}

\section{INTRODUCTION}

Safety Injection Tank (SIT) with Fluidic Device (FD) has been one of the important safety features in Advanced Power Reactors of 1400MWe (APR1400), which was designed to provide a longer injection than the existing SITs to enhance the safety for Large Break Loss-of-Coolant Accident (LBLOCA) by changing the injected flow through the FD and the standpipe of the SIT [1]. The FD has a mixing chamber to combine the flow through the standpipe and the one through the connecting holes, in which the hydraulic head and the flow rate of each flow path are balanced with interaction. As a result, the high flow injection phase and the subsequent low flow one can be achieved as longer than the existing accumulator (Fig. 1).

In the course of design and licensing reviews of APR1400 plant, extensive studies to resolve the several concerns related to the SIT hydrodynamics have been conducted [2-5]. The present paper discusses the major concerns related to SIT hydrodynamics and the direction to resolution. Modelling of SIT/FD by total hydraulic resistances, potential of nitrogen intrusion, and effect of nitrogen on LBLOCA are discussed.

\section{MODELLING OF SIT/FD}

The SIT/FD should be modelled consistent with the syntax of the applied accident analysis code such as RELAP5 [6] and MARS-KS [7] since it is an important part of the calculation of LBLOCA of the APR1400. Traditionally, the 'accum' component in both codes has been used for the SIT in the existing nuclear power plants which has no special device such as FD. Simplified one-dimensional momentum equation for an isothermal water and energy balance between water and nitrogen gas have been considered in the 'accum' component model. 
Although this simple model may provide a reasonable prediction of the water discharged from SIT for the existing NPP, it may cause a problem in prediction of nitrogen release through the standpipe during discharge phase of APR1400 (Bang et al., [4]). The reason for such a prediction was the assumption of separation of gas phase and liquid phase and the absence of specific model regarding gas entrainment by water.

To recover this problem, a generalized model using a 'pipe' component in the both codes that can consider the two phase flow of water and noncondensible gas is developed in the present study. Especially, a scheme of separate modelling of two flow paths (via the standpipe and via connecting holes) is adopted to avoid any problem induced by a model lumping the two flow paths. For this purpose, the hydraulic resistance (form loss factors, K) at each of the two flow paths should be determined for high flow phase and low flow phase, respectively. From the previous experimental study (Song et al., [8]), it was observed that the water level in the standpipe decreased rapidly during transition from the high flow phase to low flow phase and the water level of the standpipe was maintained at a value near to zero throughout the low flow phase. Such a feature important to the hydraulic resistance is considered.

\subsection{Hydraulic resistance}

Based on Fig. 1, a continuity equation and Bernoulli equations for the flow path 1 (through standpipe), the flow path 2 (through connecting holes of FD) and the overall single flow path are considered.

$$
\begin{gathered}
A_{B 1} v_{B 1}+A_{B 2} v_{B 2}=A_{B} v_{B} . \\
P_{A} / \rho g+v_{A 1}^{2} / 2 g+L_{1}=P_{B} / \rho g+v_{B}^{2} / 2 g+\left(K_{1}\right) v_{B 1}^{2} / 2 g . \\
P_{A} / \rho g+v_{A 2}^{2} / 2 g+L_{2}=P_{B} / \rho g+v_{B}^{2} / 2 g+\left(K_{1}\right) v_{B 2}^{2} / 2 g .
\end{gathered}
$$
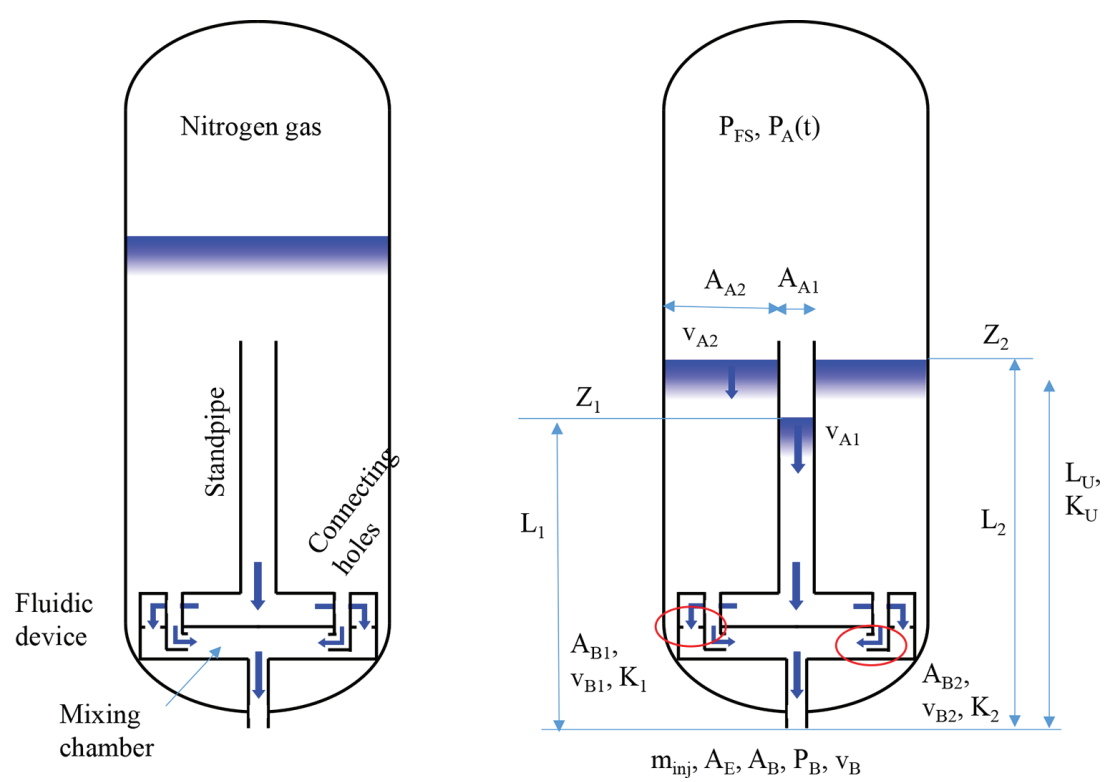

Figure 1: Configuration of SIT with FD and notation. 


$$
P_{A} / \rho g+v_{A}^{2} / 2 g+L_{U}=P_{B} / \rho g+v_{B}^{2} / 2 g+\left(K_{U}\right) v_{B}^{2} / 2 g .
$$

From the condition that the total pressure drop is the same for each flow path, we can get the following equation.

$$
\left[\left(v_{B}^{2}-f_{1}\right) K_{U} / K_{1}\right]^{1 / 2} A_{B 1}+\left[\left(v_{B}^{2}-f_{2}\right) K_{U} / K_{2}\right]^{1 / 2} A_{B 2}=v_{B} A_{B} .
$$

where,

$$
\begin{aligned}
& f_{1}=-\left(v_{A}^{2}-v_{A 1}^{2}\right)+2 g\left(L_{1}-L_{U}\right) . \\
& f_{2}=-\left(v_{A}^{2}-v_{A 2}^{2}\right)+2 g\left(L_{2}-L_{U}\right) .
\end{aligned}
$$

For the period that the water level is higher than the top of the standpipe (i.e. high flow period), the free surface should be kept. Thus, $v_{A I}=v_{A 2}=v_{A}, L_{1}=L_{2}=L_{U}$, and $f_{1}=f_{2}=0$, the eqn (3) leads to:

$$
A_{B} /\left(K_{U}\right)^{1 / 2}=A_{B 1} /\left(K_{1}\right)^{1 / 2}+A_{B 2} /\left(K_{2}\right)^{1 / 2} .
$$

For the low flow period, $f_{2}=0$, and $\left(1 / K_{1}\right)^{1 / 2}=0$, the eqn (3) can be simplified.

$$
K_{2}=\left(A_{B 2} / A_{B}\right)^{2} K_{U} \text {. }
$$

The K-factors should be determined using eqn (5) for high flow phase and eqn (6) for low flow period.

Figure 2 shows a plot of $K_{U}$ which was estimated from the measured data of SIT blowdown test of SKN unit 3 [9]. As shown in the figure, the overall K-factor shows a typical trend



Figure 2: Estimated overall K-factor. 
Table 1: Summary of hydraulic resistance in SIT.

\begin{tabular}{|c|c|c|c|c|c|c|}
\hline Case & $K_{U H}$ & $K_{U L}$ & Local $K$ & High flow & Transition $\left(0.5<\mathrm{Y}_{23}<2\right)$ & Low flow \\
\hline \multirow{4}{*}{$\begin{array}{l}\text { Lower } \\
\text { bound } \\
\text { Mean }\end{array}$} & \multirow[t]{2}{*}{10} & \multirow[t]{2}{*}{80} & $K_{1}$ & 8.33 & \multirow{3}{*}{$2.56 * Y_{23}+10.76$} & \multirow{4}{*}{$\begin{array}{l}\text { infinite } \\
12 \\
\text { infinite }\end{array}$} \\
\hline & & & $K_{2}$ & 30.3 & & \\
\hline & 20 & 100 & $K_{l}$ & 16.66 & & \\
\hline & \multirow{3}{*}{30} & \multirow{3}{*}{120} & $K_{2}$ & 60.62 & $11.15 * Y_{23}+9.48$ & \\
\hline \multirow{2}{*}{$\begin{array}{l}\text { Upper } \\
\text { bound }\end{array}$} & & & $K_{1}$ & 25 & & infinite \\
\hline & & & $K_{2}$ & 90.9 & $19.747 * \mathrm{Y}_{23}+8.2$ & 18 \\
\hline
\end{tabular}

Note: $\mathrm{Y}_{23}$ is an accumulated water level from the bottom of the standpipe

changing from low value period (high flow phase) to high value one (low flow phase) except the initial decreasing period which is corresponding to SIT motor operated valve (MOV) opening phase. From this data, mean, lower bound and upper bound of $K_{U}$ can be obtained over time interval of high flow phase and low flow phase, respectively. Then, $K_{1}$ and $K_{2}$ can be determined. Table 1 shows a result of the K-factors obtained by the process.

In this table, a formula of $K_{2}$ during a transition phase was proposed. The formula assigned for each case was based on the water level accumulated from the bottom of the standpipe. Especially, we assumed the hydraulic resistance in high flow phase water was maintained until the level in standpipe reached to $2 \mathrm{~m}$ from the bottom and the transition was ended to the low flow resistance until $0.5 \mathrm{~m}$ in the level. The basis for this assumption from the observation of the experiment.

\subsection{Calculation of SIT blowdown test}

Using the $K_{1}$ and $K_{2}$, SIT blowdown test of SKN unit 3 was calculated using MARS-KS code. Figure 3 shows a nodalization diagram to calculate the test. The $K_{1}$ and $K_{2}$, are applied to the valve 593 and junction 597. As mentioned before, valve 597 is fully closed for low flow phase. Simulation of the test was conducted by null transient for $22.5 \mathrm{~s}$ and the subsequent transient run by opening the MOV 576.

As shown in the Fig. 4, a rapid depressurization and the subsequent stable phase were observed both in test data and calculation. A good agreement between the calculation and test data was found. A little under-prediction of pressure during the initial phase is considered due to a lack of modelling of the opening characteristics of the MOV. Also, one can find the pressure curves calculated by the lower bound $\mathrm{K}$ and upper bound $\mathrm{K}$ effectively bounded the trend of the test data. Thus, the current range of $\mathrm{K}$ can be used as uncertainty range of hydraulic resistance of the SIT.

Figure 5 shows a comparison of nitrogen mass within the SIT calculated by the three cases above. The first release of nitrogen in about $40 \mathrm{~kg}$ was predicted during 60 to $80 \mathrm{~s}$ for three cases. The difference in timing of release can be reasoned for the time the water level reached the top of standpipe. The second large release of nitrogen gas was predicted at the time of SIT empty (200 300 s).

\section{CALCULATION OF LBLOCA}

Implementing the hydraulic resistance and the related modelling scheme of SIT discussed in previous chapter, calculation of a LBLOCA of APR1400 plant was conducted. The break was 


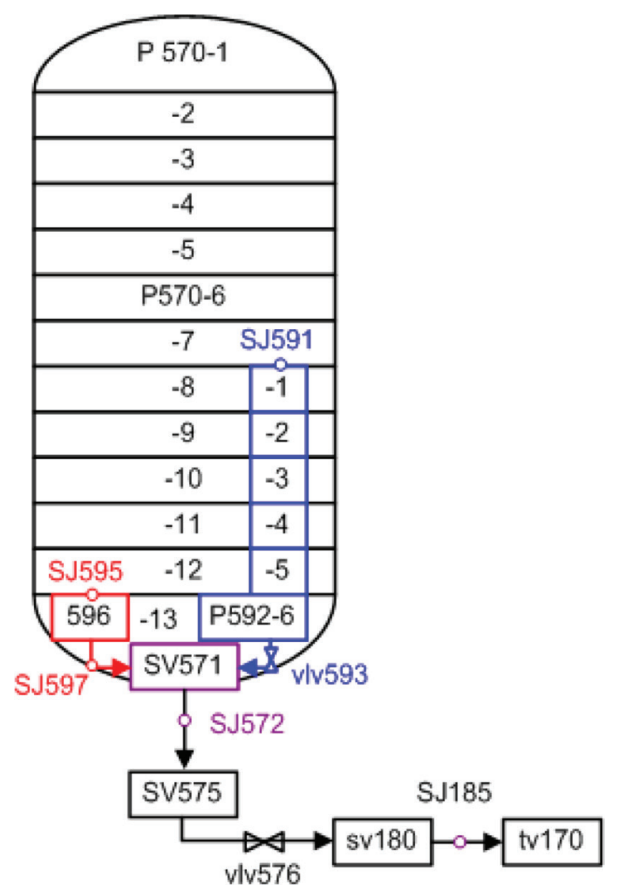

Figure 3: Nodalization of SIT/FD.

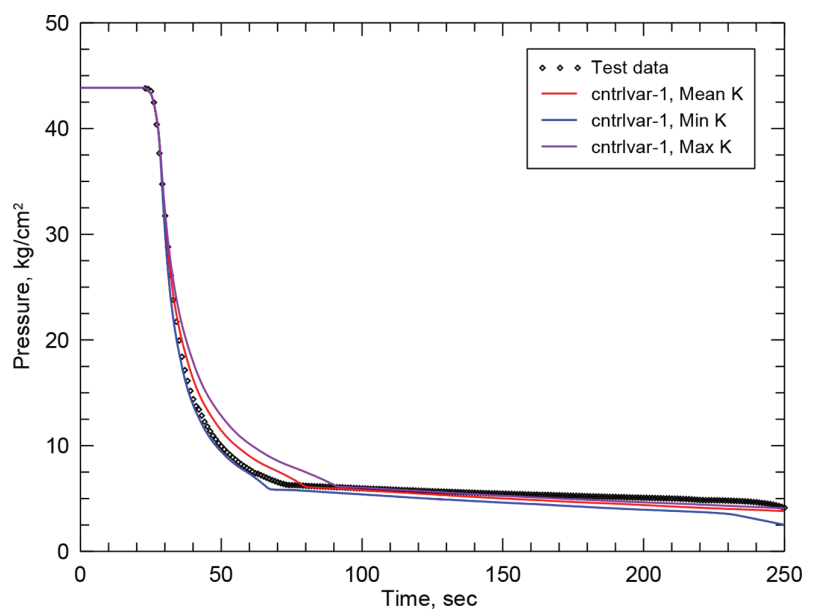

Figure 4: Pressure response.

assumed at the pump discharge side of cold leg, in double-ended guillotine shape. Figure 6 shows a MARS-KS nodalization for LBLOCA calculation, where reactor vessel, part of four cold legs, Emergency Core Cooling System (ECCS) including four SITs and Safety Injection Pumps (SIP) can be found. Two among the four SIPs were assumed to be available for the accident. Six parallel channels with crossflow junctions in azimuthal direction were used to simulate the downcomer of reactor vessel. The ECCS in Direct Vessel Injection (DVI) type is connected to the upper part of the downcomer as shown in the figure. 


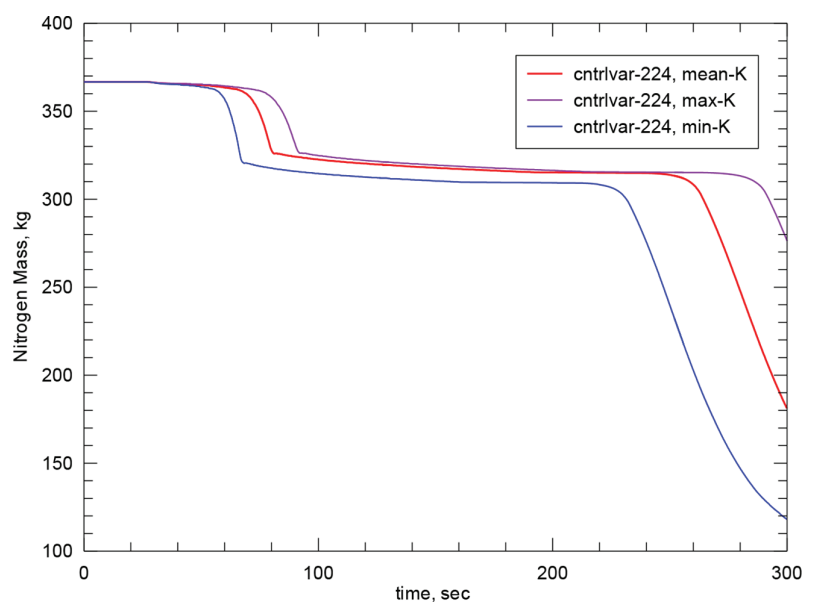

Figure 5: Comparison of nitrogen mass in SIT.
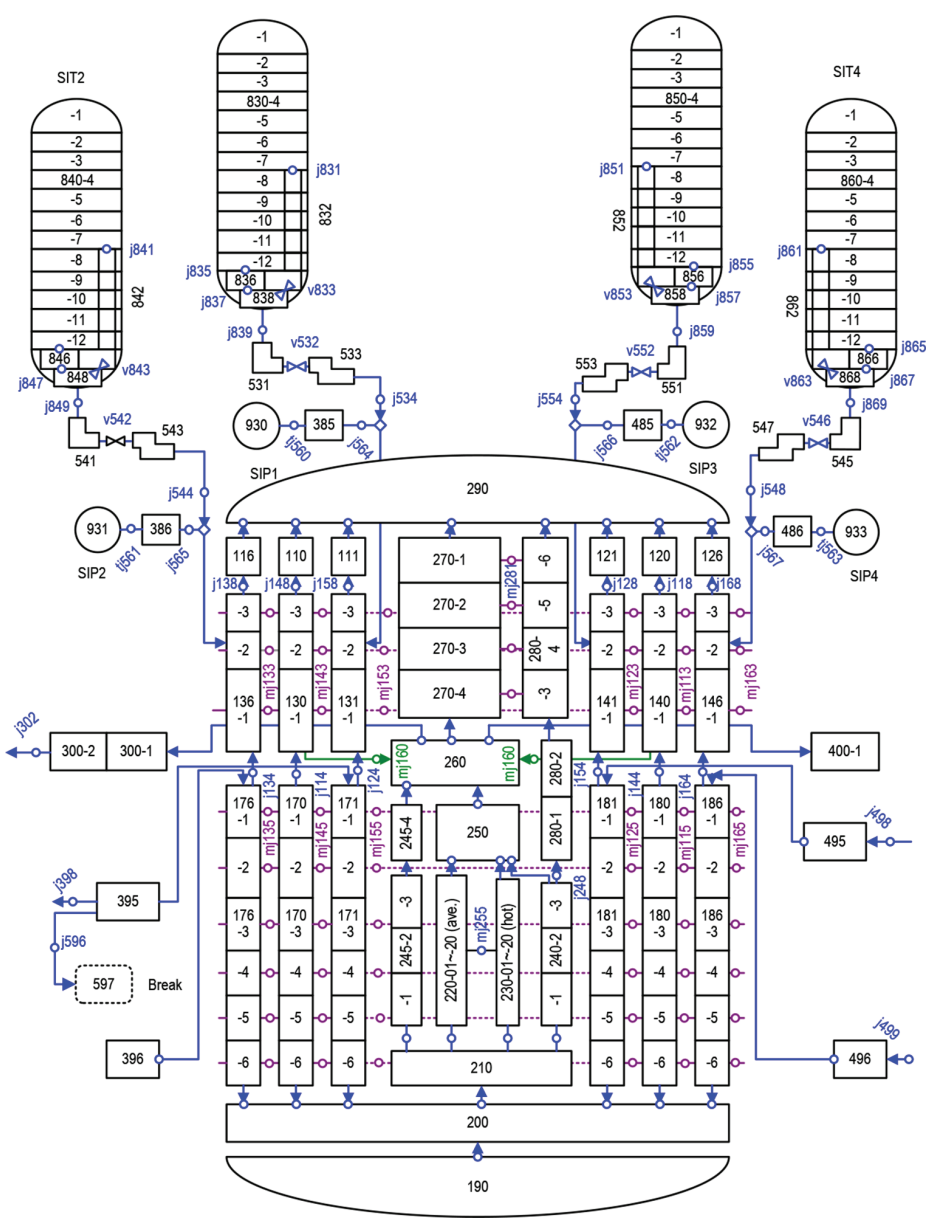

Figure 6: MARS Nodalization for APR1400 LBLOCA 


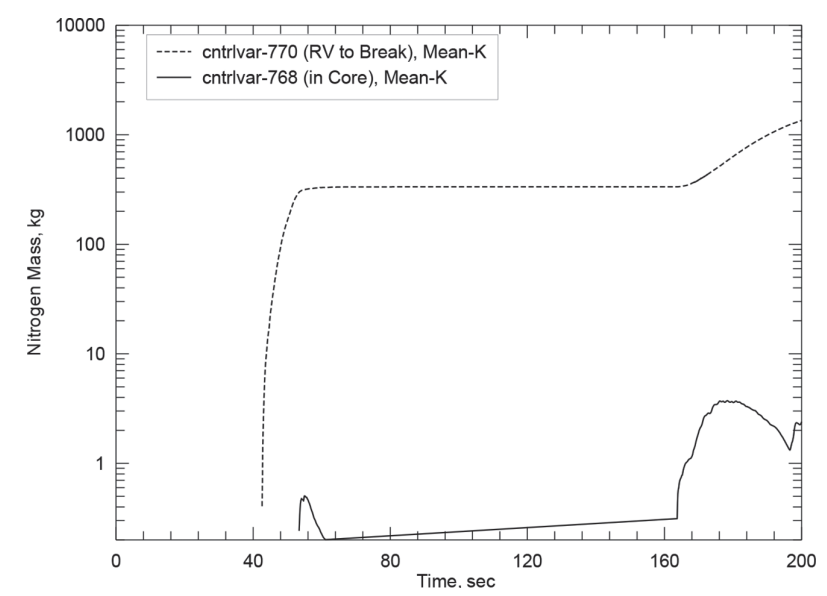

Figure 7: Comparison of nitrogen mass in core and break.

Figure 7 shows a comparison of the nitrogen mass resident in reactor core and the one accumulated at the break among the one discharged from the SIT for the mean-K case. As shown in the figure, nitrogen gas was started to introduce to the core from $40 \mathrm{~s}$ and the maximum amount of mass reached about $3 \mathrm{~kg}$. The intrusion time was almost corresponding to the transition phase from high flow to low flow of SIT. Also, one can find that total released nitrogen during transition phase was more than $200 \mathrm{~kg}$, while only a few kilograms of nitrogen were introduced to the core. It can be mentioned to due to the excessive ECCS bypass, which indicated the strong flow towards the break swept the nitrogen gas out to the break.

Figure 8 shows a comparison of fuel cladding temperature for three cases (mean, lower bound and upper bound K) following LBLOCA. Three curves show almost the same trend

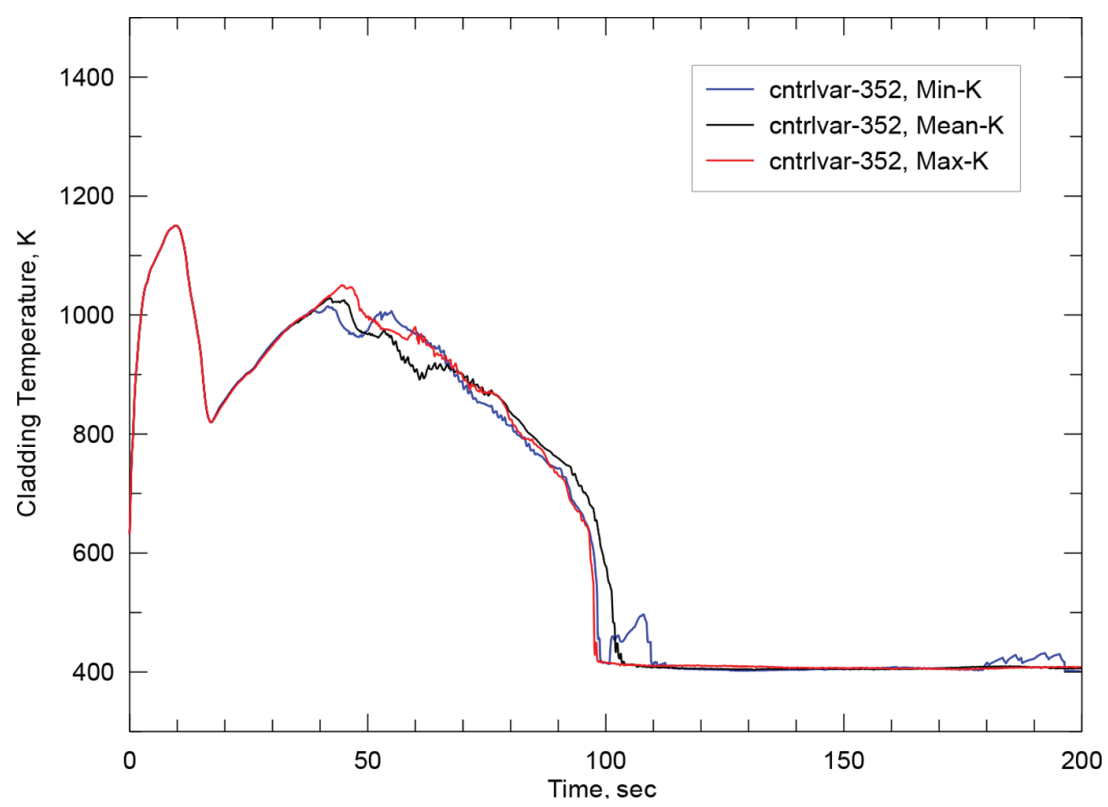

Figure 8: Comparison of cladding temperatures for three cases. 
before $40 \mathrm{~s}$, while trends after that time were deviated significantly. The difference in peak cladding temperature (PCT) between the maximum-K case and minimum-K case was higher than $40 \mathrm{~K}$, which was related to the interaction of nitrogen with water level in complex manner. Thus, the time of nitrogen intrusion could be one of the important factors to PCT.

Figure 9 shows a comparison of nitrogen mass in the core for the three cases, which supported that the maximum-K case involving the latest intrusion of nitrogen gas led to the lowest PCT. Thus, it can be stated the later and the more nitrogen leads to the lower PCT during reflood phase, which may due to the role of nitrogen gas pushing the water level up after intrusion. Also, it may be due to involve the effect of nitrogen on the core heat transfer coefficient.

Figure 10 shows a comparison of total mass flow rate injected from the SIT for three cases. It can be stated that the range of K-factors clearly had a role to change the SIT injection mass flow rate in a manner we expected.

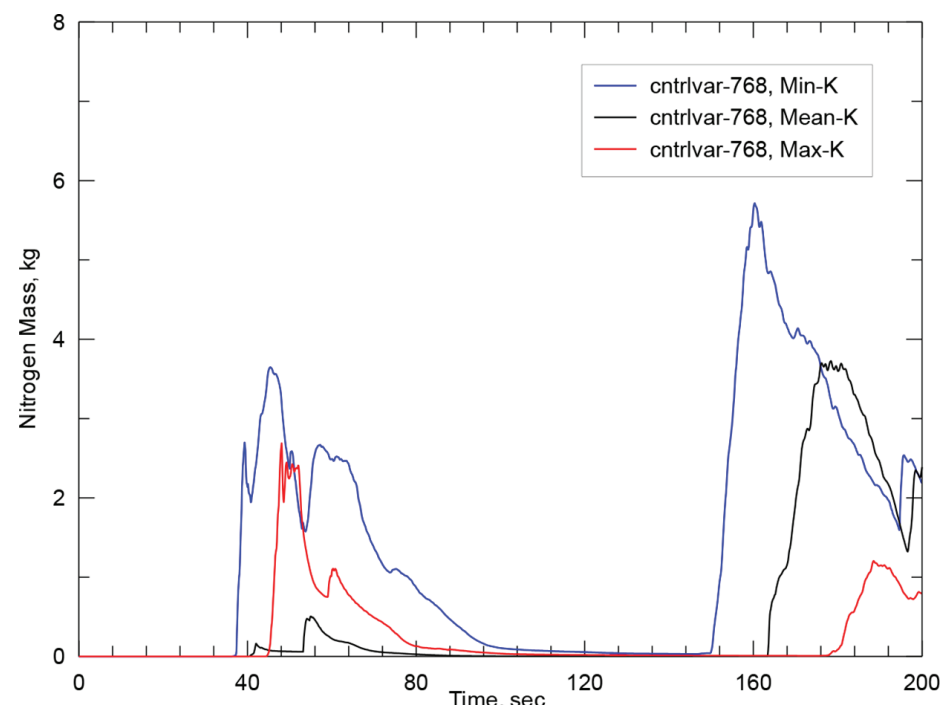

Figure 9: Comparison of nitrogen mass in core for three cases.

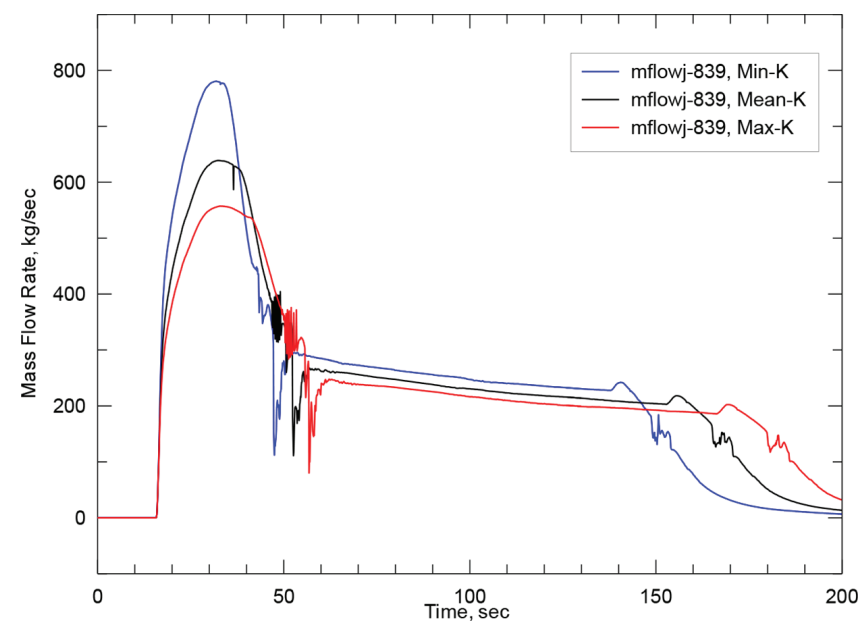

Figure 10: Comparison of mass flow rate injected from the SIT for three cases. 


\section{SUMMARY AND CONCLUSIONS}

In the present study, the effect of nitrogen gas from the SIT with FD in APR1400 nuclear power plants was discussed. To layout the amount of the nitrogen gas and timing of intrusion to the reactor core, LBLOCA calculation was conducted using the MARS-KS 1.4 code and the special SIT modelling. In the present SIT modelling, the flow path associated with the standpipe and, the flow path through the connecting holes to mixing chamber, Fluidic device and associated space were specifically considered. The most important feature of the modelling was to adopt the hydraulic resistance of the two flow paths based on the hydrodynamic consideration and the measured total loss factor. This modelling approach was validated with reasonable accuracy with a SIT blowdown test. Also, the range of uncertainty of the k-factors was derived. Through the LBLOCA calculation of APR1400, effect of nitrogen on the fuel cladding thermal response was understood. The followings can be concluded:

1. The hydraulic resistances of the standpipe flow path and the FD connecting holes path can be determined by the present scheme for high flow region and the low flow region. Also, the range of K-factors can be used as an uncertainty range for the further analysis.

2. Nitrogen gas behaviour can be predicted, and its effect on the fuel cladding thermal response can be understood through the present analysis.

3. Prediction indicated the most of the nitrogen was bypassed to the break; however, small amount of nitrogen introduced to the core made a variation of PCT in $40 \mathrm{~K}$. Thus, effect of nitrogen should be considered in a combined manner with the treatment of effect of ECCS bypass.

\section{ACKNOWLEDGEMENTS}

This work was supported by the Nuclear Safety Research Program through the Korea Foundation Of Nuclear Safety (KOFONS), granted financial resource from the Nuclear Safety and Security Commission (NSSC), Republic of Korea (No. 1305002-0416-SB120).

\section{REFERENCES}

[1] KHNP, Final Safety Analysis Report, Shinkori Units 3 and 4, KHNP, Seoul, Korea, 2015.

[2] Chu, I.C., Song, C.-H., Cho, B.H. \& Park, J.K., Development of passive flow controlling safety injection tank for APR1400. Nuclear Engineering and Design, 238(1), pp. 200-206, 2008.

https://doi.org/10.1016/j.nucengdes.2007.07.002

[3] Lim, S.G., You, S.-G. \& Kim, H-G., Numerical study of fluidic device in APR1400 using free-surface model. Transactions of the Korean Society of Mechanical Engineers B, 36(7), pp. 767-774, 2012.

https://doi.org/10.3795/ksme-b.2012.36.7.767

[4] Bang, Y.S., Lee, G.H. \& Woo, S.W., Modeling scheme of the safety injection tank with fluidic device for best estimate calculation of LBLOCA. Annals of Nuclear Energy, 75, pp. 605-610, 2015. https://doi.org/10.1016/j.anucene.2014.09.015

[5] Yoon, H.Y., Jeong, J.J., Cho, H.K., Bang, Y.S. \& Seul, K.W., A multi-scale analysis of the transient behavior of an advanced safety injection tank. Annals of Nuclear Energy, 62, pp. 17-25, 2013.

https://doi.org/10.1016/j.anucene.2013.05.042 
[6] USNRC, RELAP5/MOD3 Code Manual, NUREG/CR-5535, Washington. DC, 2001.

[7] KINS, MARS-KS Code Manual, Volume II: Input Requirements, KINS/RR-1282, Rev.1, 2016.

[8] Song C.H., Baek, W.P. \& Park, J.K., Thermal-Hydraulic R\&Ds for the APR+ developments in Korea. Nuclear Engineering and Technology, 39(4), pp. 299-312, 2007. https://doi.org/10.5516/net.2007.39.4.299

[9] KHNP, SKN 3\&4 Test Procedure (SIT Test), Rev. 1, 9P-C-441-02, Korea, 2012. 